\title{
EL VALOR DE CONOCER A ALGUIEN: GENERADOR DE RECURSOS Y EL MERCADO LABORAL EN CHILE*
}

\author{
Adolfo Fuentes \\ Centro de Estudios Públicos, Chile
}

\begin{abstract}
RESUMEN: Este artículo estudia la influencia del generador de recursos - un componente del capital social — en la probabilidad de tener un empleo y en los salarios obtenidos por los individuos, utilizando la base de datos de la encuesta Casen 2017. A partir de 11 preguntas del módulo de "Identidad, redes y participación", se construye un índice de generador de recursos. Para medir el impacto de dicho índice en el empleo se utiliza un modelo probit, mientras que para medir el impacto en los salarios se utiliza un modelo de mínimos cuadrados en dos etapas. Adicionalmente se realizan regresiones de cuantiles para determinar variaciones del efecto a lo largo de la distribución de ingresos. Los resultados obtenidos muestran que el índice está asociado positivamente con el poseer un trabajo, siendo el efecto máximo de $12,6 \%$ y $8,6 \%$ para hombres y mujeres, respectivamente. Adicionalmente, se identifica que el impacto máximo en salarios es de 18,6\% a nivel agregado; así también, el impacto del índice de generador de recursos es mayor en aquellos pertenecientes a la parte baja del I decil, y a los deciles V a IX.

PALABRAS ClAVE: generador de recursos, mercado laboral, empleo, salarios, Casen
\end{abstract}

RECIBIDO: mayo 2019 / ACEPTADO: septiembre 2019

Adolfo Fuentes es ingeniero comercial y magíster en Economía de la Universidad de Chile. Investigador asistente en el Centro de Estudios Públicos (CEP). Email: afuentes@cepchile.cl.

* Agradezco los valiosos comentarios de Ricardo González y Esteban Muñoz, así como de los árbitros anónimos de Estudios Públicos. Cualquier error u omisión son de mi exclusiva responsabilidad. 


\title{
THE VALUE OF PERSONAL CONNECTIONS: RESOURCE GENERATOR AND LABOR MARKET IN CHILE
}

\begin{abstract}
This article studies the influence of the resource generator - a component of the social capital - on the probabilities of being hired and the wages obtained by individuals, using the database of the 2017 Casen survey. ${ }^{1}$ Based on 11 questions from the "Identity, networks and participation" module, a resource generator index has been created. A probit model is used to measure the impact of the index on employment, whereas to measure the impact on wages, a two-stage least squares model is used. Additionally, quantile regressions are used to determine variations of the long-term effect on income distribution. The results obtained show that the index is positively associated with having a job, the maximum effect being $12.6 \%$ for men and $8.6 \%$ for women respectively. Also, it is noted that the impact of the maximum resource generator index on wages is $18.6 \%$ on an aggregate level. Likewise, the impact of the resource generator index is greater on individuals belonging to the lower part of the decile I, and deciles V through IX.
\end{abstract}

Received: May 2019 / AcCePted: September 2019

\section{INTRODUCCIÓN}

a importancia de las redes personales en el empleo y salarios obtenidos está ampliamente documentada. Hay artículos que ilustran cómo las redes influyen en las condiciones que permiten participar del mercado laboral (Stoloff et al. 1999; Aguilera 2002; Luke y Munshi 2006), en los mecanismos para encontrar empleo (Mouw 2003; Caliendo et al. 2011; Cingano y Rosolia 2012) y en los salarios obtenidos (Montersen y Vishwanath 1994; Sylos 2005; Delattre y Sabatier 2007; Long et al. 2017; Contreras et al. 2019). Si bien la economía ha abordado la utilización de las redes personales desde una perspectiva racional, esto es, en cuanto son la solución a un problema de asimetrías de información en el mercado laboral (Rees y Schultz 1970; Arrow y Borzekowski 2004), la influencia de las redes puede generar que individuos similares en características observables presenten diferencias salariales significativas (Montgomery 1991, 1994; Stupnytska y Zaharieva 2017) y que se produzcan problemas de eficiencia a raíz de individuos que obtienen empleos en sectores donde no tienen una ventaja competitiva (Bentolila et al. 2010; Horvarth 2014).

\footnotetext{
${ }^{1}$ National Socioeconomic Appraisal Survey.
} 
Lo anterior sugiere que estudios sobre empleabilidad y salarios que no incorporen aspectos de redes personales, están omitiendo un componente relevante. Este criterio también se puede extender hacia la medición de pobreza. El año 2015, el Ministerio de Desarrollo Social y Familia (MDSF) incorporó a la medida de pobreza multidimensional la dimensión de "Redes de apoyo y cohesión social", que tiene una ponderación de $10 \%$ del total, y se compone de tres indicadores igualmente ponderados: "Apoyo y participación social", "Seguridad" y "Trato igualitario" (Observatorio Social 2016). En particular, resulta interesante el indicador de "Apoyo y participación social", ya que está compuesto por distintas preguntas que miden si miembros del hogar conocen a personas fuera de este, que puedan ayudar en determinadas tareas y si los miembros del hogar participan en determinadas organizaciones sociales. La primera medida de este componente - conocer a personas que puedan ayudar con determinadas labores - es conocida en la literatura académica como generador de recursos (van der Gaag y Snijders 2005).

Sin embargo, a pesar de la amplia literatura existente al respecto, hay escasa evidencia para Chile que relacione el generador de recursos con resultados obtenidos en el mercado laboral. De tal forma, el objetivo de este artículo es aportar a cerrar esta brecha. En particular, se pretende dar respuesta a las siguientes preguntas: ¿cuál es el efecto de el generador de recursos en la participación laboral y en los salarios en Chile? y ¿es dicho efecto sensible al nivel de salario de las personas?

Para ello, utilizaremos los datos de la Encuesta de Caracterización Socioeconómica Nacional (Casen) del año 2017. Dicha encuesta, realizada a nivel de hogares y con representatividad nacional, cuenta entre otros con los módulos de "Registro de residentes", "Educación", "Trabajo", "Ingresos" e "Identidad, redes y participación", por lo que entrega información suficiente para estimar el impacto de dichas variables en los resultados obtenidos por cada individuo en el mercado laboral.

Para representar el generador de recursos, se construirá un índice que tomará en cuenta todas las preguntas asociadas al conocimiento de alguien que pueda ayudar en alguna labor, y la fiabilidad de dicho índice será evaluada a través del alfa de Cronbach (Cronbach 1951). Para responder a la pregunta del impacto de dicho índice en la tenencia de empleo se utilizará un modelo probit, que incorporará a las variables usuales que han sido utilizadas en Chile (Fuentes et al. 2005; Jáuregui 
2016; Fuentes y Vergara 2018a), aquella de generador de recursos. Sobre el impacto de las redes en los salarios se estimarán ecuaciones de Mincer (Mincer 1974), incorporando las correcciones de sesgo de selección (Heckman 1979) a través del método de mínimos cuadrados en dos etapas (MC2E). Para medir la influencia del generador de recursos para los distintos niveles de ingreso se estimarán regresiones de cuantiles (Koenker y Bassett 1978). Dicho método ha sido utilizado en diversas estimaciones de diferencias de pagos entre sectores productivos (Mueller 1998; Melly 2005; Fuentes y Vergara 2018b) y entre géneros (García et al. 2001; Fitzenberger y Wunderlich 2003; Gardeazabal y Ugidos 2005; Barnet-Verzat y Wolff 2008); sin embargo, no ha sido utilizado para medir impactos del generador de recursos, ya que por lo general los trabajos que estudian este componente cuentan con bases de datos más bien pequeñas, o bien, con medidas de ingreso que no permiten llegar a esta aproximación.

Cabe destacar que este artículo solo estimará el efecto del generador de recursos, omitiendo otras medidas de capital social que son relevantes en el mercado laboral, como el tamaño de las redes y el prestigio profesional (Contreras et al. 2019), ya que la encuesta Casen no cuenta con dicha información. No obstante, el aporte de este estudio es que al utilizar una encuesta de hogares se puede caracterizar correctamente el entorno socioeconómico en el que se desenvuelve cada miembro de este, y con ello se puede controlar por el sesgo de selección respecto de la participación laboral, al que están sometidos aquellos estudios que utilicen datos a nivel de individuos.

Los principales resultados obtenidos corresponden a que el índice de generador de recursos correlaciona positivamente con el poseer un trabajo. El efecto máximo del generador de recursos (al pasar de 0 a 1 ) corresponde a $12,6 \%$ y $8,6 \%$ para hombres y mujeres, respectivamente. Además, el efecto máximo del generador de recursos en los salarios es de $18,6 \%$. Por último, se identifica un efecto heterogéneo del generador de recursos para los distintos niveles deciles, ya que aquellos en la primera parte del I decil y en los deciles VI a X presentan mayores retornos que aquellos que están ubicados en los deciles II y III.

Lo anterior lleva a una discusión respecto de la relevancia de estos resultados, como la diferencia del retorno al generador de recursos por género, y a la posibilidad de establecer diferencias por fortalezas de lazos en estudios posteriores. 
La estructura de este artículo es la siguiente. En la segunda sección se revisa la literatura existente sobre los efectos de las redes en el mercado laboral, tanto en participación como en salarios. La tercera sección describe la base de datos con la que se trabaja, y además se entregan algunas estadísticas descriptivas. En la cuarta sección se expone la metodología a seguir para cada una de las preguntas que plantea este texto. La quinta sección presenta los resultados obtenidos, mientras que en la sexta sección estos son discutidos. Por último, la séptima sección presenta las conclusiones del artículo.

\section{MARCO CONCEPTUAL}

Este artículo está inserto en un área común entre la sociología y la economía; por lo tanto, para demarcar su alcance correctamente es necesario dar un marco conceptual que aborde tanto el desarrollo de la teoría de capital social en el mercado laboral desde la sociología como la estimación de ecuaciones de participación laboral y de salarios por parte de la economía.

En sociología, el capital social puede ser entendido de diversas formas; sin embargo, para efectos de este artículo seguiremos la línea de Lin (1995, 2001), Tardos (1996), Burt (1997), Portes (1998) y Völker y Flap (1999), según la cual se entiende el capital social de un individuo como aquellos recursos que están ligados a la red social (personas o asociaciones) que rodea a dicho individuo y a los mecanismos que este utiliza para beneficiarse de su uso. Por lo tanto, una condición inherente de este capital social es que los recursos no pertenecen a la persona que pretende usarlos, ${ }^{2}$ sino que esta los toma 'prestados' desde su red. Dado que hay un costo asociado al 'prestar', en la red se genera un comportamiento dinámico, en el cual las voluntades, reciprocidades y confianzas determinan las distintas cantidades de capital social que acumula cada individuo (Uehara 1990).

Sobre la importancia de la cercanía, Granovetter (1973, 1974, 1983) argumenta que los lazos débiles, esto es, aquellas relaciones más lejanas, son las más beneficiosas para el individuo en términos del mercado laboral. La razón de esto no es que el lazo sea débil per se, sino

${ }^{2}$ Esto es una diferencia fundamental que lo distingue, por ejemplo, del capital humano. 
que lazos débiles hacen más probable que una persona se conecte a otra que no conoce. De esta forma, los lazos débiles pueden ser entendidos como puentes entre dos personas (Stoloff et al. 1999; Lin 2001).

La red, como canal de información, ha sido ampliamente estudiada en la literatura que relaciona capital social con mercado laboral. Una parte de los artículos se enfoca en estudiar cómo la utilización de dichas redes puede permitir obtener un empleo. Para ello se dividen los canales de búsqueda de empleo en dos: formales e informales (Rees y Schultz 1970; Uehara 1990; Mortensen y Vishwanath 1994). Mientras los canales formales comprenden avisos en medios de comunicación y las postulaciones son directas a los empleadores a través de procesos que son públicos, los canales informales se refieren a la utilización de recomendaciones de trabajadores dentro de la organización. Algunos estudios incluso dividen el canal informal entre dos tipos de contactos, familiares y amigos, por un lado, y contactos profesionales por el otro (Sylos 2005; Bentolila et al. 2010), siguiendo la aproximación de Granovetter $(1973,1983) .^{3}$

Por otro lado, también existe una aproximación contraria a la de Granovetter, la cual postula que son los lazos fuertes aquellos capaces de activar de mejor forma el capital social. En esta línea se puede encontrar a Bourdieu (1980, 1983), Coleman (1990), y Portes y Sensenbrenner (1993), quienes argumentan que factores como la densidad de la red, la constante interacción, la reciprocidad y la confianza son factores relevantes en la activación del capital social. Sin embargo, Lin (2001) argumenta que en redes donde los individuos tengan altos niveles de confianza y cohesión, es probable que se observe que dichos individuos tengan acceso a recursos similares y, por lo tanto, la red será poco útil en dar acceso a recursos nuevos (lo que tiene relación con el principio de la homofilia). ${ }^{4}$ De esta forma, en lo respectivo a la obtención de empleos, los lazos que son considerados preponderantes son los lazos débiles. Sin embargo, hay otras áreas como el bienestar físico y

\footnotetext{
${ }^{3}$ Lo anterior es relevante para Chile ya que de, acuerdo con los datos entregados por la Encuesta "Percepciones sobre las relaciones laborales y equidad en Chile", publicados en agosto de 2008, los canales informales son preferentemente utilizados en Chile. De hecho, un $54,5 \%$ de los ocupados respondió en dicha encuesta que encontró su trabajo actual debido a amistades o familiares.

${ }^{4}$ Esto es, que las interacciones sociales tienden a tener lugar entre individuos con estilos de vida y situaciones socioeconómicas similares.
} 
psicológico (Moore y Kawachi 2017) o la participación laboral femenina (Stoloff et al. 1999), en las cuales los lazos fuertes sí son relevantes; por lo tanto, hay que tener en consideración que, de acuerdo con el área en cuestión, los lazos relevantes pueden cambiar.

Adicionalmente, hay una rama de la literatura que estudia los procesos a través de los cuales los individuos movilizan e invierten recursos con el fin de obtener ganancias de estatus (Lin 2001). Este paradigma considera que la posición social en la que se encuentra el individuo juega un rol relevante en las redes que puede generar y en el beneficio que puede extraer de ellas, por lo que individuos en mejores posiciones y con redes más diversas pueden lograr mayores retornos de su capital social. ${ }^{5}$ De esta forma, cambios en el empleo propio o ayudar a otros a conseguir empleo modifican la posición social del individuo, por lo que su estatus es variable. Esto es lo que ha derivado en el paradigma de la 'consecución de estatus' ${ }^{6}$ desarrollado inicialmente por Blau y Duncan (1967). La conclusión fundamental de este paradigma es que el estatus adquirido (nivel educacional y posiciones ocupacionales) es el factor principal para determinar el nivel de estatus de un individuo y, por tanto, este tiene mayor importancia que otros tipos de estatus como el adscrito (estatus de los padres o familia) (Lin 1999, 2001). Un estudio reciente que estudia este paradigma con datos de Chile es Contreras et al. (2019).

No obstante, los efectos de las redes no solo se relacionan con estatus o participación laboral, sino también con la desigualdad. DiMaggio y Garip (2012) proponen un mecanismo para ligar ambos conceptos. Este consiste en que, si las redes influyen, el comportamiento de los individuos y la probabilidad de adoptar una conducta está asociada a la cantidad de miembros de la red que ya la adoptaron; entonces, los individuos tenderán a agruparse entre aquellos que adoptan la conducta y entre aquellos que no lo hacen (generando homofilia). En el momento en que la conducta está correlacionada con ingresos (e.g. obtener un título universitario, trabajar en un sector), las redes pueden operar como mecanismos que contribuyen a la desigualdad económica.

\footnotetext{
${ }^{5}$ Esto es lo que también se denomina Matthew effect (Merton 1968).

${ }^{6}$ Status attainment en inglés.
} 
Adicionalmente, se han observado diferencias en la frecuencia de uso de las redes y en la composición de estas para algunos grupos, principalmente para mujeres e inmigrantes, lo que hace que obtengan retornos menores (e.g. salarios) que el resto de la población (Petersen et al. 2000; Munshi 2003; Battu et al. 2011; Beggs y Hurlbert 1997). Lo anterior es particularmente relevante en el caso chileno, donde las mujeres están expuestas a penalizaciones en carreras feminizadas (Schurch 2013), los roles de género afectan fuertemente en la decisión de participar en el mercado del trabajo (Contreras y Plaza 2010; Contreras et al. 2012) e, incluso, hay organizaciones en donde las mujeres son abiertamente menospreciadas (Mora y Blanco 2017).

Expuesto lo anterior, es posible abordar ahora específicamente el generador de recursos. En van der Gaag y Snijders (2005) se propone una forma de cuantificar variadas medidas de capital social: prestigio y educación, habilidades políticas y financieras, habilidades personales, y soporte personal. Estas medidas son obtenidas en base a preguntar al encuestado si conoce a alguien que cumpla una determinada característica y qué tipo de relación tiene con dicha persona (si es familiar, amigo o conocido). Así, por ejemplo, conocer a alguien con educación universitaria es un componente relacionado con la categoría de prestigio y educación. Conocer a alguien que conozca sobre regulación gubernamental está asociado con la categoría de habilidades políticas y financieras. Conocer a alguien que pueda hablar en un idioma extranjero corresponde a la categoría de habilidades personales. Finalmente, conocer a alguien que recomiende al encuestado para un trabajo es parte del componente de soporte personal.

Sin embargo, al momento de generar una jerarquía para evaluar la importancia del lazo, los autores no toman posición sobre cuáles lazos son los principales; esto, debido a que ello depende principalmente del objeto de estudio de aquellos lazos que son más relevantes. De esta forma, los autores trabajan el índice considerando solamente la presencia o no de lazo, y no la fortaleza de este. Esta misma posición será la utilizada para la creación del índice de generador de recursos.

Por lo tanto, este artículo utilizará preguntas que midan el generador de recursos al que tengan acceso los individuos para construir un indicador que lo represente, y para utilizarlo posteriormente en ecuaciones de participación laboral y de salarios. Es importante recalcar que este indicador no debe ser asociado directamente con capital social, 
pues constituye solo una de las distintas métricas con las que se mide dicho concepto.

Con respecto a la economía, una discusión inicial es acerca de la racionalidad de la utilización de canales informales para la búsqueda de empleados. Rees (1966) y Rees y Schultz (1970) estudian el mercado laboral en Chicago, e identifican que para los empleos de alta calificación cerca de la mitad de las contrataciones se realiza a través de los canales informales, mientras que en los empleos de baja calificación dicha proporción supera los cuatro quintos. Sin embargo, los autores no critican la utilización de estos conductos en desmedro de los canales formales, más bien la defienden. El principal argumento para la utilización de redes informales en la contratación es que, a diferencia de mercados de commodities en donde el producto a adquirir está altamente estandarizado, el mercado laboral engloba a postulantes a empleos que, a pesar de tener características estandarizables (e.g. certificados educacionales), tienen diferencias sustantivas que no son observables (e.g. productividad y motivación), y los canales informales cumplen el rol de proveer información sobre dichas diferencias entre los postulantes. Así, el uso de estos disminuye la asimetría de información entre empleador y postulante $\mathrm{y}$, por lo tanto, la utilización de canales informales por los empleadores es parte de una conducta racional (Arrow y Borzekowski 2004).

Sin embargo, esto no siempre genera resultados eficientes. Si entendemos que la productividad de los trabajadores solo es distinguible a través de sus redes, aquellos trabajadores con redes más extensas pueden presentarse como más productivos que otros con iguales niveles de productividad, pero con redes más pequeñas (Montgomery 1991, 1994; Stupnytska y Zaharieva 2017), contribuyendo a aumentar la desigualdad de ingresos. La situación anterior también es factible entre individuos de distintas productividades y distintas extensiones de redes, según lo cual aquellos de redes más extendidas pueden obtener trabajos en donde no tienen una ventaja competitiva (Bentolila et al. 2010; Horvarth 2014). Por lo tanto, las redes pueden ser entendidas como mecanismos para reducir las asimetrías de información entre candidato y empleador, pero también como mecanismos de generación de desigualdad.

Por otro lado, la economía ha medido el impacto de distintas variables sobre los salarios a través de ecuaciones de Mincer (Mincer 1974), 
en donde el salario es una función lineal de distintas variables, entre las que destacan el nivel educacional, la experiencia laboral potencial ${ }^{7}$ y el género. Adicionalmente, posteriores refinaciones incluyen variables dicotómicas para identificar premios o castigos salariales a determinadas características, como el ser inmigrante o participar en un determinado sector productivo (Borjas 2000, 2014).

Sin embargo, las mujeres enfrentan condiciones de salarios de reserva $^{8}$ diferentes de los hombres, las cuales se pueden acentuar en función de su estado civil (Mincer 1962). Dado lo anterior, estimar ecuaciones de salarios considerando únicamente a aquellos que están participando en el mercado laboral entrega coeficientes sesgados para las distintas variables. De lo anterior surge el método de corrección de Heckman $(1979,1980)$, según el cual se propone que, para estimar una ecuación de salarios, primero se debe considerar un modelo que determine la probabilidad de un individuo de tener trabajo y, posteriormente, incluir dicha medida en una ecuación de salarios tradicional. Lo mencionado se puede estimar a través del método de MC2E, o bien, a través del método de máxima verosimilitud.

En Chile, la metodología antes descrita ha sido frecuentemente utilizada con diferencias en las variables utilizadas y adaptadas a los datos disponibles (Fuentes et al. 2005; Bravo et al. 2007; Perticará y Bueno 2009; Jáuregui 2016; Fuentes y Vergara 2018a).

No obstante, este tipo de metodologías asignan la misma ganancia salarial por una característica a todos los individuos de la distribución, por lo que la metodología no permite que algunos se beneficien más que otros según su posición en la distribución de ingreso laboral. De aquí el hecho de que a estos análisis se hayan incluido las regresiones de cuantiles (Koenker y Bassett 1978), que precisamente permiten estimar distintos coeficientes a partir de la posición que se ocupe dentro de la distribución. Estas regresiones se han utilizado principalmente para determinar los efectos de premios salariales por sector o brechas de género a través de las distribuciones (Mueller 1998; García et al. 2001; Fitzenberger y Wunderlich 2003; Melly 2005; Gardeazabal y Ugidos 2005; Barnet-Verzat y Wolff 2008; Fuentes y Vergara 2018b).

\footnotetext{
${ }^{7}$ Esto es, la cantidad de años que el individuo debiese tener de experiencia laboral si es que no ha enfrentado períodos de cesantía.

${ }^{8}$ Esto corresponde al salario a partir del cual se está dispuesto a trabajar.
} 


\section{DATOS}

La base de datos que se utiliza corresponde a la encuesta Casen del año 2017. Dicha encuesta es realizada a nivel de hogares y cuenta con representatividad nacional. La encuesta posee diversos módulos entre los que destacan: "Registro de residentes", "Educación", "Trabajo", "Ingresos" e "Identidad, redes y participación", de forma tal que la encuesta provee información suficiente para realizar estimaciones tradicionales de ecuaciones de participación y de salarios, pero adicionalmente también para generar un indicador de generador de recursos por hogar.

El apartado de "Registro de residentes" permite saber cuántos miembros residen en el hogar, sus edades y géneros, además de sus lazos con el(la) jefe(a) del hogar. Por su parte, el módulo de "Educación" entrega información sobre el nivel educacional de los individuos, el cual separaremos en 13 categorías: ${ }^{9}$ sin educación, educación básica, educación media técnico-profesional, educación media científico-humanista, educación técnica de nivel superior, educación universitaria y estudios de posgrado.

De los módulos "Trabajo" e "Ingresos" se extrae información acerca de la condición laboral de los distintos miembros del hogar, las horas trabajadas, además de informar los ingresos del trabajo de aquellos que estén ocupados. Los ingresos del trabajo serán estudiados a través del logaritmo de salario por hora.

Sobre el módulo "Identidad, redes y participación" hay una serie de 11 preguntas que mide el generador de recursos. Como se mencionó en el marco conceptual, este estudio está enfocado en medir el impacto del generador de recursos entre personas que poseen acceso a recursos y entre aquellas que no, de forma que se construirá para cada pregunta un indicador dicotómico que tomará el valor 1 si alguien del hogar conoce a alguien que pueda ayudar en dicha tarea, independientemente de si esta persona vive o no en el hogar, ${ }^{10}$ y el valor 0 si es que no conoce o no sabe.

\footnotetext{
${ }^{9}$ Los niveles educacionales distintos a 'sin educación' cuentan con la diferenciación entre completa e incompleta.

${ }^{10}$ Esto incluye a la categoría 'ambas'.
} 
Sin embargo, es relevante mencionar que las preguntas sobre el generador de recursos del módulo "Identidad, redes y participación" son contestadas por un informante idóneo; por lo tanto, no es evidente que dicho individuo tenga conciencia sobre todos los generadores de recursos del hogar. De esta forma, un supuesto relevante del artículo es que los informantes idóneos conocen suficientemente bien los generadores de recursos de los demás miembros del hogar.

La Tabla 1 detalla las distintas preguntas y muestra la distribución de personas que conocen a alguien que los pueda ayudar con las diversas tareas.

A continuación, se construye el índice para medir el generador de recursos (denotado por $\alpha$ ). Este índice es el promedio simple de las 11 preguntas involucradas, de forma que posee un rango entre 0 y 1 , siendo 0 el no conocer a alguien que pueda ayudar en alguna área, y 1 el

Tabla 1. DISTRIBUCIÓN DE PORCENTAJE DE PERSONAS QUE SÍ CONOCEN A ALGUIEN, POR PREGUNTA, SEGÚN POBLACIÓN

\begin{tabular}{|c|c|}
\hline Pregunta: ¿Alguien de su hogar conoce a alguien que... & $\begin{array}{l}\text { Porcentaje } \\
\text { que si conoce }\end{array}$ \\
\hline $\begin{array}{l}\text {...pueda ayudar en el cuidado en caso de enfermedad de algún } \\
\text { miembro del hogar? }\end{array}$ & 89,7 \\
\hline $\begin{array}{l}\text {...pueda ayudar en el cuidado de niños(as) o personas en } \\
\text { situación de discapacidad o dependientes del hogar? }\end{array}$ & 84,9 \\
\hline ...pueda facilitar un vehículo si el hogar lo necesita? & 84,6 \\
\hline ...pueda prestar dinero al hogar en caso de emergencia? & 78,4 \\
\hline $\begin{array}{l}\text {...pueda ayudar al hogar a resolver consultas o realizar trámites } \\
\text { legales o financieros? }\end{array}$ & 73,2 \\
\hline $\begin{array}{l}\text {...pueda ayudar al hogar en el uso de tecnologías (computador, } \\
\text { internet, etc.)? }\end{array}$ & 85,8 \\
\hline $\begin{array}{l}\text {...pueda ayudar con reparaciones del hogar (gasfiteria, } \\
\text { mecánica, albañilería o artículos eléctricos)? }\end{array}$ & 79,4 \\
\hline $\begin{array}{l}\text {...pueda ayudar a conseguir trabajo a algún miembro del hogar } \\
\text { (servir de referencias, información o contratar)? }\end{array}$ & 61,4 \\
\hline $\begin{array}{l}\text {...pueda aconsejar a los miembros del hogar en caso de } \\
\text { problemas personales o familiares? }\end{array}$ & 83,3 \\
\hline ...pueda hablar o escribir en otro idioma? & 48,9 \\
\hline ...tenga educación profesional universitaria? & 71,9 \\
\hline
\end{tabular}

Fuente: elaboración propia sobre la base de Casen 2017. 
conocer a personas que puedan ayudar en todas las áreas. Dicha escala es consistente internamente, ya que la fiabilidad obtenida a través del Alpha de Cronbach corresponde a 0,83. Adicionalmente, el índice presenta una media de 0,77 y una desviación estándar de 0,25.

Los gráficos 1 y 2 muestran la frecuencia absoluta y acumulada para dicho índice. Como se puede observar, la distribución no es simétrica, concentrándose la mayor masa en la parte derecha de la distribución, con un $27 \%$ de personas que presentan un índice de 1 , mientras que solo un 3\% presenta un índice de 0 . Adicionalmente, un $41 \%$ de la población conoce a personas que puedan ayudar en siete áreas o menos, mientras que el $59 \%$ restante conoce a personas que las pueden ayudar, al menos, en ocho áreas.

Adicionalmente, es interesante estudiar la relación que presenta el índice con el nivel de ingreso, lo que está representado en la Tabla 2. Como se puede observar, el índice es consistentemente creciente en ingreso.

Gráfico 1. FRECUENCIA ABSOLUTA DEL GENERADOR DE RECURSOS

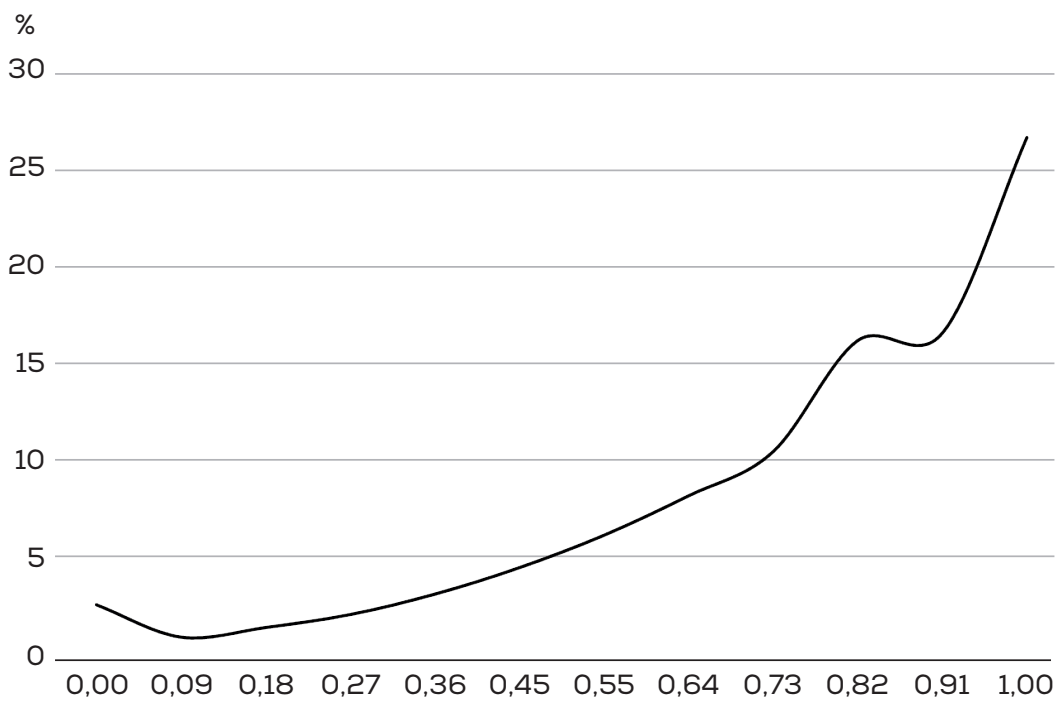

Fuente: elaboración propia sobre la base de Casen 2017. 
Gráfico 2. FRECUENCIA ACUMULADA DEL GENERADOR DE RECURSOS

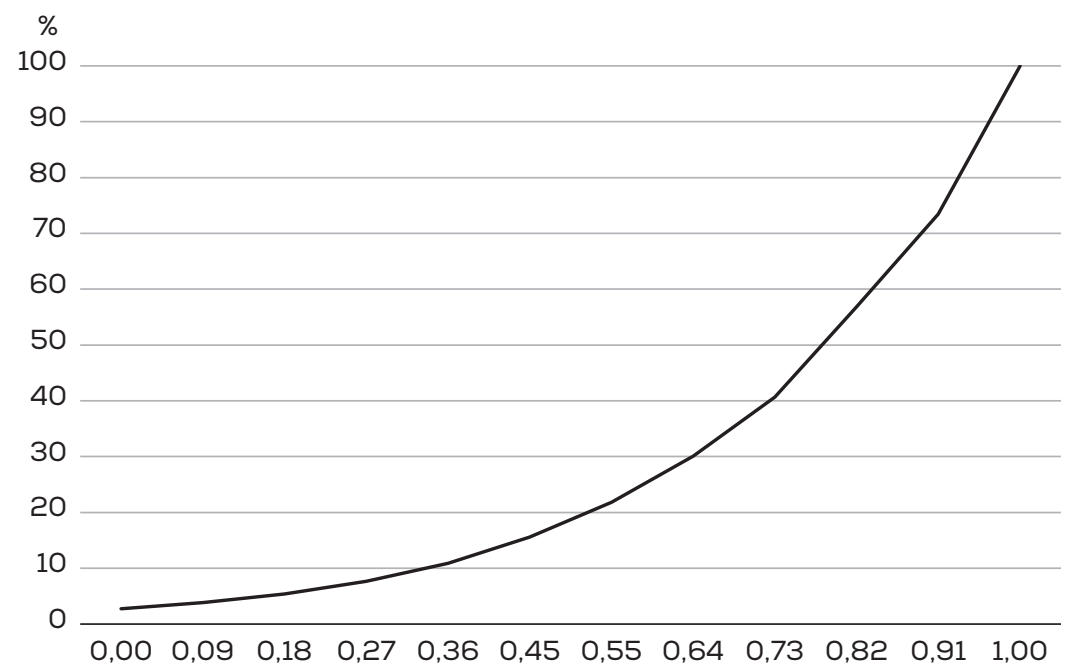

Fuente: elaboración propia sobre la base de Casen 2017.

Tabla 2. MEDIA E INTERVALO DE CONFIANZA DEL ÍNDICE DE GENERADOR DE RECURSOS

\begin{tabular}{lccc}
\hline Decil & Media indice & \multicolumn{2}{c}{ Intervalo de confianza 95\% } \\
\hline I & 0,678 & 0,668 & 0,689 \\
II & 0,715 & 0,705 & 0,724 \\
III & 0,733 & 0,723 & 0,743 \\
IV & 0,738 & 0,728 & 0,747 \\
V & 0,752 & 0,742 & 0,762 \\
VI & 0,773 & 0,764 & 0,783 \\
VII & 0,788 & 0,778 & 0,797 \\
VIII & 0,808 & 0,798 & 0,817 \\
IX & 0,846 & 0,836 & 0,856 \\
X & 0,890 & 0,879 & 0,901 \\
\hline
\end{tabular}

Fuente: elaboración propia sobre la base de Casen 2017.

La Tabla 3 presenta una estadística descriptiva de las variables que se utilizarán para los cálculos de los modelos de participación. En dicha base fueron excluidas aquellas personas menores de 15 años y mayores de 64. La razón de esto es que los menores de 15 años no pueden participar en el mercado laboral y las personas mayores de 64 años que participen de dicho mercado es probable que tengan características distintas a aquellas que decidieron jubilar. 
Tabla 3. ESTADÍSTICAS DESCRIPTIVAS DE LAS OBSERVACIONES A UTILIZAR EN LA ECUACIÓN DE PARTICIPACIÓN LABORAL

\begin{tabular}{|c|c|c|c|c|}
\hline & Media & Desv. est. & Min. & Máx. \\
\hline \multicolumn{5}{|l|}{ Variable dependiente } \\
\hline Posee trabajo & 0,441 & 0,497 & 0 & 1 \\
\hline \multicolumn{5}{|l|}{ Generador de recursos } \\
\hline Alpha & 0,771 & 0,242 & 0 & 1 \\
\hline \multicolumn{5}{|l|}{ Variables demográficas } \\
\hline Edad & 38,404 & 14,560 & 15 & 64 \\
\hline Edad $^{2}$ & 1686,900 & 1149,765 & 225 & 4096 \\
\hline Género (Mujer = 1) & 0,528 & 0,499 & 0 & 1 \\
\hline Jefe de hogar (Si = 1) & 0,346 & 0,476 & 0 & 1 \\
\hline $\begin{array}{l}\text { Número de personas } \\
\text { en el hogar }\end{array}$ & 3,880 & 1,658 & 1 & 19 \\
\hline $\begin{array}{l}\text { Ingreso promedio del hogar } \\
\text { (sin propio) }\end{array}$ & 11,827 & 1,080 & 2.351 & 17.551 \\
\hline Zona (Rural = 1) & 0,183 & 0,387 & 0 & 1 \\
\hline $\begin{array}{l}\text { Cantidad de menores de } 6 \\
\text { años en el hogar }\end{array}$ & 0,345 & 0,610 & 0 & 6 \\
\hline \multicolumn{5}{|l|}{ Variables educacionales } \\
\hline \multicolumn{5}{|l|}{ Sin educación } \\
\hline Ed. básica incompleta & 0,093 & 0,291 & 0 & 1 \\
\hline Ed. básica completa & 0,094 & 0,292 & 0 & 1 \\
\hline $\begin{array}{l}\text { Ed. media técnico-profesional } \\
\text { incompleta }\end{array}$ & 0,024 & 0,153 & 0 & 1 \\
\hline $\begin{array}{l}\text { Ed. media técnico-profesional } \\
\text { completa }\end{array}$ & 0,082 & 0,275 & 0 & 1 \\
\hline $\begin{array}{l}\text { Ed. media científico-humanista } \\
\text { incompleta }\end{array}$ & 0,133 & 0,339 & 0 & 1 \\
\hline $\begin{array}{l}\text { Ed. media cientifico-humanista } \\
\text { completa }\end{array}$ & 0,245 & 0,430 & 0 & 1 \\
\hline $\begin{array}{l}\text { Ed. técnica nivel superior } \\
\text { incompleta }\end{array}$ & 0,037 & 0,189 & 0 & 1 \\
\hline $\begin{array}{l}\text { Ed. técnica nivel superior } \\
\text { completa }\end{array}$ & 0,068 & 0,252 & 0 & 1 \\
\hline Ed. universitaria incompleta & 0,089 & 0,285 & 0 & 1 \\
\hline Ed. universitaria completa & 0,101 & 0,301 & 0 & 1 \\
\hline
\end{tabular}




\begin{tabular}{|c|c|c|c|c|}
\hline $\begin{array}{l}\text { Estudios de posgrado } \\
\text { incompletos }\end{array}$ & 0,003 & 0,057 & 0 & 1 \\
\hline $\begin{array}{l}\text { Estudios de posgrado } \\
\text { completos }\end{array}$ & 0,010 & 0,100 & 0 & 1 \\
\hline \multicolumn{5}{|l|}{ Variables estado civil } \\
\hline Casado(a) & 0,310 & 0,462 & 0 & 1 \\
\hline Conviviente o pareja sin $\mathrm{AUC}^{*}$ & 0,180 & 0,384 & 0 & 1 \\
\hline Conviviente o pareja con AUC & 0,002 & 0,050 & 0 & 1 \\
\hline Anulado(a) & 0,001 & 0,032 & 0 & 1 \\
\hline Separado(a) & 0,049 & 0,216 & 0 & 1 \\
\hline Divorciado(a) & 0,017 & 0,129 & 0 & 1 \\
\hline Viudo(a) & 0,016 & 0,124 & 0 & 1 \\
\hline Soltero(a) & 0,425 & 0,494 & 0 & 1 \\
\hline \multicolumn{5}{|l|}{ Variables zona geográfica } \\
\hline Tarapacá & 0,047 & 0,211 & 0 & 1 \\
\hline Antofagasta & 0,040 & 0,197 & 0 & 1 \\
\hline Atacama & 0,031 & 0,174 & 0 & 1 \\
\hline Coquimbo & 0,044 & 0,206 & 0 & 1 \\
\hline Valparaiso & 0,089 & 0,285 & 0 & 1 \\
\hline $\begin{array}{c}\text { Libertador Gral. Bernardo } \\
\text { O'Higggins }\end{array}$ & 0,073 & 0,260 & 0 & 1 \\
\hline Maule & 0,070 & 0,256 & 0 & 1 \\
\hline Bio-Bio & 0,100 & 0,300 & 0 & 1 \\
\hline La Araucanía & 0,070 & 0,256 & 0 & 1 \\
\hline Los Lagos & 0,057 & 0,233 & 0 & 1 \\
\hline Gral. Carlos Ibáñez del Campo & 0,024 & 0,152 & 0 & 1 \\
\hline Magallanes y Antártica chilena & 0,032 & 0,175 & 0 & 1 \\
\hline Metropolitana de Santiago & 0,202 & 0,401 & 0 & 1 \\
\hline Los Rios & 0,047 & 0,212 & 0 & 1 \\
\hline Arica y Parinacota & 0,035 & 0,183 & 0 & 1 \\
\hline Nuble & 0,038 & 0,191 & 0 & 1 \\
\hline Observaciones & \multicolumn{4}{|c|}{139,967} \\
\hline
\end{tabular}

Nota: * Acuerdo de unión civil.

Fuente: elaboración propia sobre la base de Casen 2017.

Finalmente, la Tabla 4 presenta una estadística descriptiva respecto de las variables a utilizar para los modelos de salarios. Dicha base posee una menor cantidad de observaciones dado que enfrenta la condición de tener un trabajo. 
Tabla 4. ESTADÍSTICAS DESCRIPTIVAS DE LAS OBSERVACIONES A UTILIZAR EN LA ECUACIÓN DE SALARIOS

\begin{tabular}{|c|c|c|c|c|}
\hline & Media & Desv. est. & Min. & Máx. \\
\hline \multicolumn{5}{|l|}{ Variable dependiente } \\
\hline Logaritmo salario por hora & 7,754 & 0,681 & 3.912 & 13.933 \\
\hline \multicolumn{5}{|l|}{ Generador de recursos } \\
\hline Alpha & 0,783 & 0,237 & 0 & 1 \\
\hline \multicolumn{5}{|l|}{ Inverso del ratio de Mills } \\
\hline Lambda & 0,706 & 0,371 & 0 & 2.902 \\
\hline \multicolumn{5}{|l|}{ Variables demográficas } \\
\hline Género $($ Mujer $=1)$ & 0,443 & 0,497 & 0 & 1 \\
\hline \multicolumn{5}{|l|}{ Variables laborales } \\
\hline Experiencia laboral potencial & 21,678 & 13,720 & 0 & 58 \\
\hline Experiencia laboral potencial $^{2}$ & 658,146 & 670,063 & 0 & 3364 \\
\hline \multicolumn{5}{|l|}{ Variables educacionales } \\
\hline Sin educación & 0,005 & 0,072 & 0 & 1 \\
\hline Ed. básica incompleta & 0,074 & 0,262 & 0 & 1 \\
\hline Ed. básica completa & 0,086 & 0,280 & 0 & 1 \\
\hline $\begin{array}{l}\text { Ed. media técnico-profesional } \\
\text { incompleta }\end{array}$ & 0,014 & 0,116 & 0 & 1 \\
\hline $\begin{array}{l}\text { Ed. media técnico-profesional } \\
\text { completa }\end{array}$ & 0,101 & 0,301 & 0 & 1 \\
\hline $\begin{array}{l}\text { Ed. media cientifico-humanista } \\
\text { incompleta }\end{array}$ & 0,087 & 0,281 & 0 & 1 \\
\hline $\begin{array}{l}\text { Ed. media cientifico-humanista } \\
\text { completa }\end{array}$ & 0,264 & 0,441 & 0 & 1 \\
\hline $\begin{array}{l}\text { Ed. técnica nivel superior } \\
\text { incompleta }\end{array}$ & 0,036 & 0,185 & 0 & 1 \\
\hline Ed. técnica nivel superior completa & 0,099 & 0,298 & 0 & 1 \\
\hline Ed. universitaria incompleta & 0,054 & 0,227 & 0 & 1 \\
\hline Ed. universitaria completa & 0,158 & 0,364 & 0 & 1 \\
\hline Estudios de posgrado incompletos & 0,006 & 0,074 & 0 & 1 \\
\hline Estudios de posgrado completos & 0,018 & 0,132 & 0 & 1 \\
\hline \multicolumn{5}{|l|}{ Variables rama ocupacional } \\
\hline $\begin{array}{l}\text { Agricultura, ganadería, caza y } \\
\text { silvicultura }\end{array}$ & 0,108 & 0,310 & 0 & 1 \\
\hline Pesca & 0,013 & 0,112 & 0 & 1 \\
\hline Explotación de minas y canteras & 0,031 & 0,174 & 0 & 1 \\
\hline Industrias manufactureras & 0,078 & 0,268 & 0 & 1 \\
\hline
\end{tabular}




\begin{tabular}{|c|c|c|c|c|}
\hline $\begin{array}{l}\text { Suministro de electricidad, gas y } \\
\text { agua }\end{array}$ & 0,011 & 0,104 & 0 & 1 \\
\hline Construcción & 0,083 & 0,276 & 0 & 1 \\
\hline $\begin{array}{l}\text { Comercio al por mayor y al por } \\
\text { menor }\end{array}$ & 0,145 & 0,352 & 0 & 1 \\
\hline Hoteles y restaurantes & 0,047 & 0,211 & 0 & 1 \\
\hline $\begin{array}{c}\text { Transporte, almacenamiento y } \\
\text { comunicación }\end{array}$ & 0,064 & 0,245 & 0 & 1 \\
\hline Intermediación financiera & 0,016 & 0,125 & 0 & 1 \\
\hline $\begin{array}{l}\text { Actividades inmobiliarias, } \\
\text { empresariales }\end{array}$ & 0,061 & 0,238 & 0 & 1 \\
\hline Administración pública y defensa & 0,075 & 0,263 & 0 & 1 \\
\hline Enseñanza & 0,108 & 0,310 & 0 & 1 \\
\hline Servicios sociales y de salud & 0,066 & 0,249 & 0 & 1 \\
\hline $\begin{array}{l}\text { Otras actividades de servicios } \\
\text { comunitarios }\end{array}$ & 0,027 & 0,162 & 0 & 1 \\
\hline $\begin{array}{l}\text { Hogares privados con servicio } \\
\text { doméstico }\end{array}$ & 0,056 & 0,230 & 0 & 1 \\
\hline Otro & 0,011 & 0,106 & 0 & 1 \\
\hline \multicolumn{5}{|l|}{ Variables zona geográfica } \\
\hline Tarapacá & 0,045 & 0,208 & 0 & 1 \\
\hline Antofagasta & 0,038 & 0,192 & 0 & 1 \\
\hline Atacama & 0,029 & 0,167 & 0 & 1 \\
\hline Coquimbo & 0,038 & 0,192 & 0 & 1 \\
\hline Valparaiso & 0,091 & 0,288 & 0 & 1 \\
\hline $\begin{array}{c}\text { Libertador Gral. Bernardo } \\
\text { O'Higggins }\end{array}$ & 0,080 & 0,272 & 0 & 1 \\
\hline Maule & 0,072 & 0,258 & 0 & 1 \\
\hline Bio-Bio & 0,094 & 0,292 & 0 & 1 \\
\hline La Araucanía & 0,061 & 0,239 & 0 & 1 \\
\hline Los Lagos & 0,056 & 0,229 & 0 & 1 \\
\hline Gral. Carlos Ibáñez del Campo & 0,025 & 0,156 & 0 & 1 \\
\hline Magallanes y Antártica chilena & 0,037 & 0,189 & 0 & 1 \\
\hline Metropolitana de Santiago & 0,225 & 0,418 & 0 & 1 \\
\hline Los Ríos & 0,043 & 0,204 & 0 & 1 \\
\hline Arica y Parinacota & 0,028 & 0,166 & 0 & 1 \\
\hline Ñuble & 0,036 & 0,187 & 0 & 1 \\
\hline Observaciones & & 61,350 & & \\
\hline
\end{tabular}

Fuente: elaboración propia sobre la base de Casen 2017. 


\section{METODOLOGÍA}

\section{Modelo de participación}

En primer lugar, se estima un modelo probit para determinar la participación en el mercado del trabajo. Dicho modelo es usual en la literatura, sin embargo, las variables que se utilizan tienden a ser diferentes.

En particular, aquí se seguirá la metodología aplicada en Fuentes et al. (2005), Jáuregui (2016) y en Fuentes y Vergara (2018b) debido a que en estos trabajos se utilizan los datos de la encuesta Casen. Así, se estima la probabilidad de trabajar para cada género por separado y se define dicha probabilidad como una función que depende de:

a) Indicador de generador de recursos

b) Edad y edad al cuadrado

c) Nivel educacional

d) Estado civil

e) Jefe de hogar (si es o no lo es)

f) Zona donde se vive (rural o no)

g) Número de personas en el hogar

h) Ingreso promedio del hogar sin considerar el del individuo a analizar $^{11}$

i) Cantidad de niños menores de 6 años en el hogar

j) Región donde se vive

Los niveles educacionales se miden en base a las siguientes categorías, considerando sus estudios completo e incompleto por separado:

- Educación básica

- Educación media técnico-profesional

- Educación media científico-humanista

- Educación técnica de nivel superior (CFT e IP) $)^{12}$

- Educación universitaria

- Estudios de posgrado

Todos estos componentes pasan a definir un vector $x$ asociado a cada individuo $i$, de un total de $n$. Si $y_{i}$ es una variable que toma los valores 1 y 0 , en función de si se observa a una persona participar del

\footnotetext{
${ }^{11}$ Por ejemplo, si estamos calculando dicho ingreso para una jefa de hogar, su ingreso es excluido.

${ }^{12}$ Centros de formación técnica (CFT) e institutos profesionales (IP).
} 
mercado laboral o no, y asumiendo una distribución normal se pueden calcular los $\beta$ que maximizan la siguiente expresión:

$$
L\left(x_{i}, \beta\right)=\prod_{i=1}^{N} \Phi\left(x_{i}^{\prime} \beta\right)^{y_{i}} \cdot\left[1-\Phi\left(x_{i}^{\prime} \beta\right)\right]^{1-y_{i}}
$$

Donde son de interés los efectos marginales asociados a dichos coeficientes, que vienen determinados por:

$$
\frac{\partial \mathrm{P}\left[\mathrm{y}_{\mathrm{i}}=1\right]}{\partial \mathrm{x}_{\mathrm{i}}^{\prime}}=\phi\left(x_{i}^{\prime} \beta\right) \cdot \beta
$$

En particular es de interés el parámetro asociado al indicador de generador de recursos.

\section{Modelo de salarios}

El modelo para determinar los salarios sigue la aproximación de Heckman (1979), en donde se debe corregir por el sesgo de selección, y se hace a través del método MC2E. Cuando se tienen los estimadores $\hat{\beta}$, se calcula la inversa del ratio de Mills, que será utilizado como proxy de la probabilidad de observar a cada individuo trabajando. Dicho parámetro se define como:

$$
\lambda_{i}=\frac{\phi\left(x_{i}^{\prime} \hat{\beta}\right)}{\Phi\left(x_{i}^{\prime} \hat{\beta}\right)}
$$

Posteriormente, se realiza una estimación de salarios por hora a través de mínimos cuadrados ordinarios, incluyendo en la estimación la inversa del ratio de Mills. En particular, se estima la siguiente ecuación:

$$
\begin{gathered}
\ln w h_{i}=\gamma_{o}+\gamma_{1} \cdot \exp _{i}+\gamma_{2} \cdot \exp _{i}^{2}+\gamma_{3} \cdot g_{i} \\
+\sum_{l=1}^{12} \gamma_{3+i} \cdot E d u c_{i, l}+\sum_{j=1}^{15} \gamma_{13+j} \cdot R_{j} \\
+\sum_{m=1}^{17} \gamma_{28+m} \cdot S_{m}+\gamma_{46} \cdot \lambda_{i}+\gamma_{47} \cdot \alpha_{i}+u_{i}
\end{gathered}
$$


Donde $\exp _{i}$ y $\exp _{i}^{2}$ denotan la experiencia laboral potencial ${ }^{13}$ y su cuadrado, respectivamente. $E d u c_{i, l}$ es una variable dicotómica que toma el valor 1 si es que el individuo $i$ posee el nivel de educación $l,{ }^{14}$ y 0 en el caso contrario. $R_{j}$ es una variable dicotómica que toma el valor $1 \mathrm{si}$ es que el individuo $i$ vive en la región $j,{ }^{15}$ y 0 en el caso contrario. $S_{m}$ es una variable que toma el valor 1 si es que el individuo $i$ trabaja en el sector $m$, y 0 en el caso contrario. Los sectores considerados son los siguientes: ${ }^{16}$

- Agricultura, ganadería, caza y silvicultura

- Pesca

- Explotación de canteras y minas

- Industrias manufactureras

- Suministro de electricidad, gas y agua

- Construcción

- Comercio al por mayor y al por menor

- Hoteles y restaurantes

- Transporte, almacenamiento y comunicaciones

- Intermediación financiera

- Actividades inmobiliarias

- Administración pública y defensa

- Enseñanza

- Servicios sociales y de salud

- Otras actividades de servicios comunitarios

- Hogares privados con servicio doméstico

- Organizaciones y órganos extraterritoriales

Finalmente, $\lambda_{i}$ corresponde a la inversa del ratio de Mills del individuo $i$, y $\alpha_{i}$ corresponde a su índice de generador de recursos.

En particular, el parámetro de interés corresponde a aquel asociado a la variable $\alpha_{i}$.

\footnotetext{
${ }^{13}$ Esta variable corresponde a la edad menos los años de escolaridad menos seis. Se utiliza como proxy de la experiencia laboral potencial.

${ }^{14}$ El nivel 'sin educación' es el nivel base.

${ }^{15} \mathrm{La}$ I región es la región base.

${ }^{16} \mathrm{El}$ sector base corresponde a 'sin dato'.
} 


\section{Regresiones de cuantiles}

El cálculo anterior representa una medida promedio del efecto del indicador de generador de recursos sobre los salarios ya que, en términos conceptuales, la metodología anterior asigna a todos los individuos el mismo coeficiente independientemente de su posición dentro de la distribución de ingresos.

Un aspecto relevante que estudiar es si el impacto del indicador de generador de recursos varía a lo largo de la distribución de ingresos, esto es, si personas de mayores ingresos se benefician de manera diferente, en términos de salario del generador de recursos, que las personas de menores ingresos.

Para ello, utilizaremos regresiones de cuantiles (Koenker y Bassett 1978). Dado un cuantil $p$ que pertenece al rango $(0,1)$, estimar una regresión de cuantiles es equivalente a resolver el problema dado por:

$$
\hat{\beta}_{p}=\arg \min \left[\sum_{i: y \geq x_{i}^{\prime} \beta} p\left|y_{i}-x_{i}^{\prime} \beta\right|+\sum_{i: y \leq x_{i}^{\prime} \beta}(1-p)\left|y_{i}-x_{i}^{\prime} \beta\right|\right]
$$

Donde el parámetro $y_{i}$ se estima a través de:

$$
y_{i}=x_{i}^{\prime} \beta+u_{p}
$$

Bajo la restricción según la cual:

$$
E\left(y_{i} \mid x_{i}\right)_{\text {Dado cuantil } p}=x_{i}^{\prime} \beta
$$

La estimación de (3) será con la misma forma funcional que usamos en (1); esto, con el propósito de obtener resultados comparables con la estimación anterior.

Estos problemas se suelen escribir como uno de programación lineal y son resueltos vía simplex (Koenker y D’Orey 1987). Para cada estimación, que está asociada a un cuantil, se obtiene un conjunto de coeficientes $\hat{\beta}_{p}$ que son válidos solo para dicho espacio de la distribución. Por lo tanto, para obtener una visión completa de la evolución de los coeficientes a lo largo de la distribución, realizaremos la estimación para los 99 percentiles. 


\section{RESULTADOS}

\section{Participación}

La Tabla 5 presenta los resultados para la submuestra de hombres. Con respecto a las variables demográficas, se obtienen coeficientes que van en línea con la literatura que analiza la participación laboral. La edad aumenta la probabilidad de trabajar, aunque a una tasa decreciente. El ser jefe de hogar no es estadísticamente significativo, así como tampoco lo es la cantidad de personas que viven en el hogar o la cantidad de niños menores de 6 años. Por otro lado, aumentos de ingresos promedio del hogar, sin considerar el propio, disminuyen la probabilidad de participar en el mercado laboral, al igual que vivir en una zona rural.

A nivel de variables educacionales, encontramos que mayores niveles de educación aumentan la probabilidad de tener un trabajo y, dentro del mismo nivel, el haber completado dicho nivel genera un mayor efecto que el no haberlo completado.

Por último, el coeficiente Alpha de generador de recursos aumenta la probabilidad de tener empleo. En particular, un hombre que conoce a alguien que pueda ayudarlo en las 11 tareas tiene un 12,6\% más de probabilidad de tener un empleo que un hombre que no conoce a nadie que pueda ayudarlo en alguna tarea.

La Tabla 6 presenta los resultados para la submuestra de mujeres. Con respecto a las variables demográficas, la edad aumenta la probabilidad de trabajar y a una tasa creciente. El ser jefa de hogar es estadísticamente significativo, a diferencia de lo que ocurre en los hombres.

Por otro lado, el número de personas en el hogar y la cantidad de menores de 6 años, afectan negativamente la probabilidad de tener empleo en las mujeres. Lo anterior, como ya había sido mencionado, tiene relación con que los roles de género son fuertes en Chile, incluso a nivel latinoamericano (Contreras et al. 2012).

A nivel de variables educacionales se encuentra, al igual que en los hombres, que mayores niveles educacionales aumentan la probabilidad de tener un empleo. Adicionalmente, para los niveles más altos de educación los efectos marginales de las mujeres son mayores a los de los hombres.

Por último, el coeficiente Alpha de generador de recursos también aumenta la probabilidad de tener empleo, aunque en menor medida que 
Tabla 5. EFECTOS MARGINALES DE MODELO PROBIT DE PARTICIPACIÓN LABORAL DE HOMBRES

\begin{tabular}{|c|c|c|}
\hline & Efecto marginal & Desv. est. \\
\hline \multicolumn{3}{|l|}{ Generador de recursos } \\
\hline Alpha & $0,126^{* \star *}$ & 0,014 \\
\hline \multicolumn{3}{|l|}{ Variables demográficas } \\
\hline Edad & $0,055^{* * *}$ & 0,001 \\
\hline Edad $^{2}$ & $-0,001^{* * *}$ & 0,000 \\
\hline Jefe de hogar $(\mathrm{Si}=1)$ & 0,017 & 0,011 \\
\hline Número de personas en el hogar & $-0,003$ & 0,003 \\
\hline Ingreso promedio del hogar (sin propio) & $-0,156^{\star \star *}$ & 0,004 \\
\hline Zona (Rural = 1) & $-0,047^{\star \star *}$ & 0,011 \\
\hline $\begin{array}{c}\text { Cantidad de menores de } 6 \text { años } \\
\text { en el hogar }\end{array}$ & $-0,008$ & 0,007 \\
\hline \multicolumn{3}{|l|}{ Variables educacionales } \\
\hline Ed. básica incompleta & $0,119^{\star \star \star}$ & 0,025 \\
\hline Ed. básica completa & $0,142^{\star \star *}$ & 0,025 \\
\hline $\begin{array}{l}\text { Ed. media técnico-profesional } \\
\text { incompleta }\end{array}$ & $0,075^{* *}$ & 0,032 \\
\hline Ed. media técnico-profesional completa & $0,263^{* * *}$ & 0,021 \\
\hline $\begin{array}{l}\text { Ed. media cientifico-humanista } \\
\text { incompleta }\end{array}$ & $0,122^{* * *}$ & 0,024 \\
\hline Ed. media científico-humanista completa & $0,251^{* \star *}$ & 0,024 \\
\hline Ed. técnica nivel superior incompleta & $0,219^{* * *}$ & 0,025 \\
\hline Ed. técnica nivel superior completa & $0,305^{\star * *}$ & 0,022 \\
\hline Ed. universitaria incompleta & $0,111^{\star \star \star}$ & 0,026 \\
\hline Ed. universitaria completa & $0,355^{\star * *}$ & 0,018 \\
\hline Estudios de posgrado incompletos & $0,349^{* * *}$ & 0,025 \\
\hline Estudios de posgrado completos & $0,385^{\star \star *}$ & 0,016 \\
\hline \multicolumn{3}{|l|}{ Controles adicionales } \\
\hline Estado civil & \multicolumn{2}{|r|}{ Si } \\
\hline Zona geográfica & \multicolumn{2}{|r|}{$\mathrm{Si}$} \\
\hline
\end{tabular}

Nota: ${ }^{* *}$ indica una significancia al $95 \%$ y ${ }^{* * *}$ al $99 \%$.

Fuente: elaboración propia sobre la base de Casen 2017. 
Tabla 6. EFECTOS MARGINALES DE MODELO PROBIT DE PARTICIPACIÓN LABORAL DE MUJERES

\begin{tabular}{|c|c|c|}
\hline & Efecto marginal & Desv. est. \\
\hline \multicolumn{3}{|l|}{ Generador de recursos } \\
\hline Alpha & $0,086^{* * *}$ & 0,015 \\
\hline \multicolumn{3}{|l|}{ Variables demográficas } \\
\hline Edad & $0,060^{* * *}$ & 0,001 \\
\hline Edad $^{2}$ & $0,000^{* * *}$ & 0,000 \\
\hline Jefe de hogar ( $\mathrm{Si}=1)$ & $0,062^{* \star *}$ & 0,008 \\
\hline Número de personas en el hogar & $-0,007^{\star \star *}$ & 0,002 \\
\hline Ingreso promedio del hogar (sin propio) & $-0,093^{* * *}$ & 0,004 \\
\hline Zona (Rural = 1) & $-0,087^{\star \star *}$ & 0,008 \\
\hline $\begin{array}{c}\text { Cantidad de menores de } 6 \text { años } \\
\text { en el hogar }\end{array}$ & $-0,007^{* * *}$ & 0,002 \\
\hline \multicolumn{3}{|l|}{ Variables educacionales } \\
\hline Ed. básica incompleta & 0,033 & 0,026 \\
\hline Ed. básica completa & $0,062^{\star *}$ & 0,026 \\
\hline $\begin{array}{l}\text { Ed. media técnico-profesional } \\
\text { incompleta }\end{array}$ & $0,056^{*}$ & 0,031 \\
\hline Ed. media técnico-profesional completa & $0,255^{* * *}$ & 0,026 \\
\hline $\begin{array}{l}\text { Ed. media cientifico-humanista } \\
\text { incompleta }\end{array}$ & $0,055^{\star *}$ & 0,027 \\
\hline Ed. media científico-humanista completa & $0,190^{* * *}$ & 0,025 \\
\hline Ed. técnica nivel superior incompleta & $0,239^{* * *}$ & 0,027 \\
\hline Ed. técnica nivel superior completa & $0,380^{* \star *}$ & 0,024 \\
\hline Ed. universitaria incompleta & $0,127^{* * *}$ & 0,027 \\
\hline Ed. universitaria completa & $0,480^{* * *}$ & 0,020 \\
\hline Estudios de posgrado incompletos & $0,496^{* * *}$ & 0,031 \\
\hline Estudios de posgrado completos & $0,541^{* * *}$ & 0,016 \\
\hline
\end{tabular}

Controles adicionales

Estado civil

Zona geográfica

Nota: * indica una significancia al $90 \%,{ }^{* *}$ al $95 \%$ y ${ }^{* * *}$ al $99 \%$.

Fuente: elaboración propia sobre la base de Casen 2017. 
en los hombres. En particular, una mujer que conoce a alguien que pueda ayudarla en las 11 tareas tiene un 8,6\% más de probabilidad de tener un empleo que una mujer que no conoce a nadie que pueda ayudarla en alguna tarea. Sin embargo, esta comparación asume que las distribuciones de los dos grupos, en particular las varianzas, son similares. Lo anterior resulta difícil de mantener debido a las percepciones del rol de la mujer, lo que será analizado con más detalle en la sección de discusión.

\section{Salarios}

La Tabla 7 presenta los resultados de la estimación de la ecuación de salarios. Al respecto de las variables laborales se estima que la experiencia laboral potencial tiene un efecto positivo sobre el salario y presenta una tasa de crecimiento positiva, aunque pequeña en magnitud. Por otro lado, el retorno a los niveles educacionales es coherente con la estimación previa: niveles más altos de educación tienen un mayor retorno educacional que los anteriores. Adicionalmente, se mantiene la diferencia entre los niveles incompletos y completos.

La variable asociada al género implica que las mujeres reciben un $18,3 \%$ menos de salario que un hombre a iguales observables, siendo una magnitud que está en línea con los otros estudios realizados (Fuentes et al. 2005; Jáuregui 2016; Fuentes y Vergara 2018b).

Por último, el coeficiente Alpha presenta un coeficiente de 18,6\%. Esto implica que, a iguales observables, un trabajador que conozca a personas que lo puedan ayudar en las 11 tareas obtiene un 18,6\% más de salario que aquel trabajador que no conoce a personas que lo puedan ayudar en alguna tarea.

\section{Cuantiles}

Sin embargo, como se había mencionado anteriormente, el efecto de $18,6 \%$ es un efecto agregado para todos los trabajadores; por lo tanto, es posible que su impacto varíe a través de la distribución de ingreso. Para obtener un indicador para cada percentil de ingreso, se realizan las regresiones de cuantiles.

El Gráfico 3 presenta la evolución del coeficiente desde el percentil 1 (aquellos de menores ingresos) al percentil 99 (aquellos de mayores ingresos). 
Tabla 7. COEFICIENTES LINEALES DE ESTIMACIÓN DE SALARIOS

\begin{tabular}{|c|c|c|}
\hline & Coeficiente & Desv. est \\
\hline \multicolumn{3}{|l|}{ Generador de recursos } \\
\hline Alpha & $0,186^{* * *}$ & 0,017 \\
\hline \multicolumn{3}{|l|}{ Variables demográficas } \\
\hline Género & $-0,183^{\star * *}$ & 0,008 \\
\hline \multicolumn{3}{|l|}{ Variables laborales } \\
\hline Experiencia laboral potencial & $0,030^{* * *}$ & 0,001 \\
\hline Experiencia laboral potencial ${ }^{2}$ & $0,000^{* * *}$ & 0,000 \\
\hline \multicolumn{3}{|l|}{ Variables educacionales } \\
\hline Ed. básica incompleta & $0,103^{*}$ & 0,056 \\
\hline Ed. básica completa & $0,143^{* *}$ & 0,057 \\
\hline $\begin{array}{l}\text { Ed. media técnico-profesional } \\
\text { incompleta }\end{array}$ & $0,255^{\star \star \star}$ & 0,065 \\
\hline Ed. media técnico-profesional completa & $0,363^{* * *}$ & 0,058 \\
\hline $\begin{array}{l}\text { Ed. media científico-humanista } \\
\text { incompleta }\end{array}$ & $0,159^{* * *}$ & 0,058 \\
\hline Ed. media cientifico-humanista completa & $0,300^{* * *}$ & 0,058 \\
\hline Ed. técnica nivel superior incompleta & $0,489^{* * *}$ & 0,059 \\
\hline Ed. técnica nivel superior completa & $0,617^{* * *}$ & 0,058 \\
\hline Ed. universitaria incompleta & $0,594^{* * *}$ & 0,060 \\
\hline Ed. universitaria completa & $1,197^{\star \star \star}$ & 0,061 \\
\hline Estudios de posgrado incompletos & $1,538^{\star \star *}$ & 0,068 \\
\hline Estudios de posgrado completos & $1,787^{* * *}$ & 0,069 \\
\hline \multicolumn{3}{|l|}{ Control de sesgo de selección } \\
\hline Lambda & $0,181^{\star \star \star}$ & 0,015 \\
\hline \multicolumn{3}{|l|}{ Controles adicionales } \\
\hline Rama ocupacional & \multicolumn{2}{|c|}{ Sí } \\
\hline Zona geográfica & \multicolumn{2}{|c|}{ Si } \\
\hline $\mathrm{R}^{2}$ & \multicolumn{2}{|c|}{0,416} \\
\hline
\end{tabular}

Nota: ${ }^{*}$ indica una significancia al $90 \%,{ }^{* *}$ al $95 \%$ y ${ }^{* * *}$ al $99 \%$.

Fuente: elaboración propia sobre la base de Casen 2017. 
Gráfico 3. ESTIMACIÓN DE EFECTO DE GENERADOR DE RECURSOS POR PERCENTIL

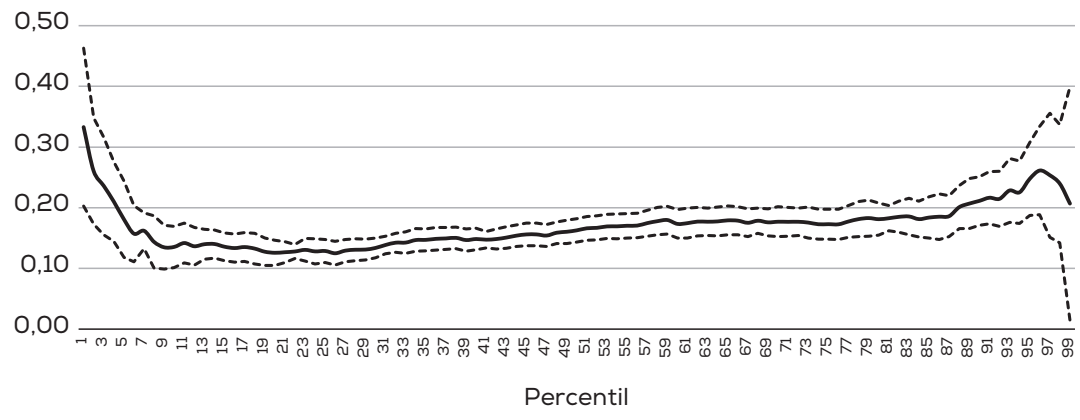

Nota: intervalos de confianza al 95\%.

Fuente: elaboración propia sobre la base de Casen 2017.

Como se puede observar, no todos los percentiles reciben el mismo beneficio en el salario al cambiar su índice de generador de recursos desde 0 a 1 . En primer lugar, el beneficio del generador de recursos es mayor en la parte inferior del I decil de ingresos, además de los deciles V a IX. Por otra parte, aquellos pertenecientes a los deciles II y III son los que reciben menores beneficios del generador de recursos.

\section{DISCUSIÓN}

Los resultados expuestos en la sección anterior son interesantes; sin embargo, es necesaria una discusión sobre su contexto, limitaciones y posibles caminos de investigación que se abren a futuro. Adicionalmente, resulta interesante analizar cómo estos resultados afectan a la política pública, en particular a aquella relacionada con la medición de carencia en redes de soporte. En primer lugar, se debe considerar que los resultados presentados corresponden a correlaciones y, por lo tanto, no hay una condición de causalidad directa entre las variables dependientes - entre ellos el generador de recursos-y las variables independientes - la participación o el salario por hora. De esta forma, el supuesto que hace este tipo de metodología es que primero se determina el generador de recursos y posteriormente se obtiene el empleo y el salario. 
A pesar de ello, es posible que la relación sea inversa, vale decir, que el empleo e ingresos de un individuo determinen al generador de recursos a los que el individuo tiene acceso. No obstante, el caso anterior no está sustentado por teoría, a diferencia de la metodología aplicada en este artículo, que está respaldada en la teoría del capital social que sigue la línea de Lin (1995, 2001), Tardos (1996), Burt (1997), Portes (1998) y Völker y Flap (1999). Lo anterior no implica que sí pueda existir una dinámica entre los dos componentes donde, por ejemplo, un determinado nivel de generador de recursos permite obtener un empleo y un salario que, a su vez, permita conocer a distintas personas que incrementen el generador de recursos del individuo, dando la posibilidad de acceder a nuevos empleos con mejores salarios. Sin embargo, para testear este tipo de comportamiento son necesarios datos de panel, y la encuesta Casen no posee dicha característica.

Además, debemos considerar que el generador de recursos es solo una de las distintas métricas que existen para medir capital social; por lo tanto, esta medida no puede ser calificada como representativa del capital social que posea un determinado individuo. Adicionalmente, si bien las preguntas planteadas en la encuesta Casen siguen la línea de van der Gaag y Snijders (2005), las respuestas no están ordenadas por cercanía de lazo (familiar, amigo, conocido), sino que están ordenadas por si viven en el hogar encuestado. Lo anterior impide que se testee con exactitud una aproximación como la de Granovetter (1973, 1974, 1983), según la cual son los lazos débiles aquellos que generan mayores retornos en términos de capital social respecto de la obtención de trabajo.

Dado que un resultado de este artículo es proveer evidencia respecto de que el generador de recursos sí influye en algunos aspectos - como la obtención de empleo y los salarios obtenidos_-, indistintamente de si dicho generador vive dentro o fuera del hogar, puede ser pertinente reconsiderar la definición de carencia utilizada por el MDSF. Esto, debido a que la carencia en la categoría de "Apoyo y participación social" viene determinada exclusivamente por la ausencia de personas fuera del hogar que puedan ayudar en las tareas mencionadas, lo cual no tiene respaldo en la literatura académica, particularmente porque esto tendería a ignorar los lazos fuertes de los individuos, los cuales, como se mencionó anteriormente, están más asociados a aspectos de salud y de participación laboral femenina. 
Para la construcción de dicho índice no se consideran tres preguntas que corresponden a conocer a alguien que: 1) "pueda ayudar en el cuidado de niños(as) o personas en situación de discapacidad o dependientes del hogar", 2) "pueda hablar o escribir en otro idioma" y 3) "tenga educación profesional universitaria". La justificación para dejar fuera la primera pregunta es que pueden existir hogares en que no sea necesario contar con dicho recurso (Observatorio Social 2016). Esto es razonable; sin embargo, lleva a preguntarse por qué se incluye una pregunta que puede medir recursos necesarios solo para determinados grupos, pues la función última del generador de recursos es generar mediciones comparables entre individuos. Las dos preguntas siguientes son eliminadas debido a que se asocian principalmente a estatus (Observatorio Social 2016), y no a recursos sociales, lo cual también es cuestionable dado que un mayor estatus puede estar ligado a acceder a una mayor diversidad de recursos sociales.

Por otra parte, si el MDSF busca caracterizar con mayor precisión las distintas redes que poseen las personas, se hace necesario modificar el cuestionario Casen e incluir el tipo de lazo que las une (e.g. familiar, amigos o conocidos). Dicha modificación permitiría entender las diferencias de generadores de recursos que hay entre los distintos lazos, estudiar cuáles pueden ser relevantes para áreas específicas (e.g. salud, empleo, educación) y analizar qué hogares son carentes de ellos.

La mayor contribución de este artículo a la literatura viene de la mano de medir el impacto de este indicador particular con una base de datos que es medida a nivel de hogares y que posee una gran cantidad de observaciones. Que sea a nivel de hogares es un factor relevante, ya que nos permite caracterizar correctamente el contexto socioeconómico en el que se desenvuelve cada persona, lo cual es fundamental para poder realizar la estimación de participación en el mercado laboral, y evitar parte del sesgo de selección al que están sometidos estudios que utilicen bases de datos a nivel individual. Por otro lado, el gran número de observaciones que posee esta encuesta permite realizar estimaciones a nivel de percentiles, obteniendo coeficientes para representar adecuadamente cada parte de la distribución.

Como es de esperar, los resultados obtenidos también presentan limitaciones. Al momento de construir el generador de recursos se asumió que los informantes idóneos conocían bien los generadores de 
recursos de los demás miembros del hogar. Si bien este puede ser un supuesto razonable, si la condición planteada no se cumple, entonces la construcción del generador de recursos puede estar sesgada, y con ello es susceptible de alterar los resultados obtenidos. El hecho de que se utilice la variable 'experiencia laboral potencial' como proxy de la experiencia laboral de ambos grupos, puede ser una buena aproximación a la experiencia laboral en hombres, pero no en mujeres, dado que enfrentan trayectorias laborales más heterogéneas. De esta forma, se puede estar sobreestimando la cantidad de experiencia laboral que poseen, lo que a su vez puede alterar los resultados obtenidos. Adicionalmente, las percepciones sobre el rol de la mujer, que para efectos de este artículo actúan como no observables, pueden sesgar los coeficientes estimados, particularmente los encontrados en la ecuación de participación laboral, ya que al realizar la comparación entre los coeficientes de los modelos probit se está asumiendo que las distribuciones y particularmente las varianzas entre los dos grupos (hombres y mujeres), son similares (Allison 1999; Williams 2009; Mood 2010). Lo anterior hace que el efecto del generador de recursos pueda no ser diferenciado por género; sin embargo, ello no sesga uno de los resultados principales de este artículo, que corresponde a que el generador de recursos tiene influencia sobre la probabilidad de estar empleado.

Otra limitación está asociada a la interpretación del coeficiente relativo al generador de recursos. En este artículo se optó por ajustar el generador para medir el efecto entre el cambio de 0 (no conocer a nadie) a 1 (conocer a alguien en todas las áreas). Si bien metodológicamente se podría estimar la ganancia salarial por cada pregunta, lo anterior no resulta prudente, ya que implícitamente se está asumiendo que cada pregunta genera el mismo aporte marginal a la empleabilidad o al salario. De esta forma, los efectos encontrados en este artículo corresponden a las diferencias máximas posibles.

Este artículo abre algunos caminos de investigación, los cuales pueden ser divididos en dos. Por un lado, resulta interesante que la brecha de género estimada en este artículo sea de 18,3\%, mientras que la encontrada en la literatura enfocada a este aspecto sea en torno a $20 \%$ (Jáuregui 2016; Fuentes y Vergara 2018b). Dado que se utilizan metodologías que siguen una estructura y variables comunes, la diferencia entre las brechas puede ser explicada por la incorporación del indicador 
de generador de recursos y, por lo tanto, se puede formular la hipótesis de que existe un beneficio diferente por género para dicha medida. Estudiar esto apuntaría a obtener evidencia que explique la brecha salarial de género que hay en Chile, a través de caminos que no han sido abordados por la literatura.

Por otro lado, se encuentra la posibilidad de realizar mediciones en función de los lazos que declaran los agentes. Este artículo fue neutro en este aspecto, estudiando el generador de recursos de forma tal de separar a aquellos que conocen o no conocen a alguien que los ayude en una determinada tarea, sin importar la fortaleza del lazo. Sin embargo, puede ser interesante realizar estudios que tomen una posición sobre la importancia del lazo para las distintas preguntas, y también observar cómo los resultados varían entre especificaciones de estos. Incluso se podrían generar escalas en donde, en determinadas preguntas, se percibiesen como más relevantes los lazos fuertes y en otras los lazos débiles.

\section{CONCLUSIONES}

Este artículo estudia la influencia del generador de recursos —un componente de capital social - en el mercado del trabajo, particularmente en el tener un empleo y en los salarios obtenidos. Para ello se utiliza la base de datos de la encuesta Casen 2017. A partir de 11 preguntas del módulo de "Identidad, redes y participación" se construye un índice de generador de recursos. Este índice está basado en la teoría que propone que el capital social puede ser entendido como los recursos a los cuales se tiene acceso a través de las redes personales. Para medir el impacto de dicho índice en el poseer un empleo, se utiliza un modelo probit, mientras que para medir el impacto en los salarios se utiliza un modelo de MC2E, que cuenta con la corrección por sesgo de selección.

Los resultados obtenidos muestran que el índice está asociado positivamente con el poseer un trabajo, aunque su efecto es diferente para hombres y mujeres, siendo el efecto máximo de $12,6 \%$ y $8,6 \%$ para cada grupo, respectivamente. Se identifica, también, que el impacto máximo en salarios es de $18,6 \%$ a nivel agregado. Por último, se encuentra que el impacto del índice de generador de recursos en la parte baja del I decil y en los deciles V a IX, es mayor al encontrado en el resto de los deciles. 
Todo lo anterior debe ser atendido en un contexto de correlación y no de causalidad, además de considerar que el generador de recursos son solo una parte del capital social. Este artículo es asimismo neutro respecto de la importancia de los lazos, de forma que se evaluó la diferencia entre aquellos que cuentan con un lazo y aquellos que no.

Los resultados anteriores abren una serie de preguntas sobre la influencia de las redes en la brecha salarial de género, además de interrogar sobre cómo la fortaleza de dichos lazos hace cambiar los resultados encontrados. Además, se propone modificar la definición de vulnerabilidad en el apartado "Apoyo y participación social", junto con medir en el cuestionario el tipo de lazo que posee cada persona frente a los distintos generadores de recursos.

\section{BIBLIOGRAFÍA}

Aguilera, M. 2002. The Impact of Social Capital on Labor Force Participation: Evidence from 2000 Social Capital Benchmark Survey. Social Science Quarterly 83(3), 853-874.

Allison, P. 1999. Comparing Logit and Probit Coefficients across Groups. Sociological Methods \& Research 28(2), 186-208.

Arrow, K. y Borzekowski, R. 2004. Limited Network Connections and the Distribution of Wages. FEDS Working Paper 2004/41.

Barnet-Verzat, C. y Wolff, F.-Ch. 2008. Gender Wage Gap and the Glass Ceiling Effect: A Firm-Level Investigation. International Journal of Manpower 29(6), 486-502.

Battu, H., Seaman, P. y Zenou, Y. 2011. Job Contact Networks and the Ethnic Minorities. Labour Economics 18(1), 48-56.

Beggs, J. y Hurlbert, J. 1997. The Social Context of Men's and Women's Job Search Ties: Membership in Voluntary Organizations, Social Resources, and Job Search Outcomes. Sociological Perspectives 40(4), 601-622.

Bentolila, S., Michelacci, C. y Suárez, J. 2010. Social Contacts and Occupational Choice. Economica 77, 20-45.

Blau, P. y Duncan, O. 1967. The American Occupational Structure. New York: John Wiley and Sons.

Borjas, G. 2000. Labor Economics. New York: Mc Graw Hill.

Borjas, G. 2014. Immigration Economics. Cambridge: Harvard University Press.

Bourdieu, P. 1980. Le capital social: notes provisoires. Actes de la Recherche en Sciences Sociales 3, 2-3.

Bourdieu, P. 1983. The Forms of Capital (241-258). En Richardson, J.G. (ed.), Handbook of Theory and Research for the Sociology of Education. Westport, CT: Greenwood. 
Bravo, D., Sanhueza, C. y Urzúa, S. 2007. Ability, Schooling Choices and Gender Labor Market Discrimination: Evidence for Chile. Serie Documentos de Trabajo 265. Departamento de Economía, Universidad de Chile.

Burt, R. 1997. The Contingent Value of Social Capital. Administrative Science Quarterly 42(2), 339-365.

Caliendo, M., Schmild, R. y Uhlendorff, A. 2011. Social Networks, Job Search Methods and Reservation Wages: Evidence for Germany. International Journal of Manpower 32(7), 796-824.

Cingano, F. y Rosolia, A. 2012. People I Know: Job Search and Social Networks. Journal of Labor Economics 30(2), 291-332.

Coleman, J. 1990. Foundations of Social Theory. Cambridge, MA: Harvard University Press.

Contreras, D. y Plaza, G. 2010. Cultural Factors in Women's Labor Force Participation in Chile. Feminist Economics 16, 27-46.

Contreras, D., Hurtado, A. y Sara, S. 2012. La excepción chilena y las percepciones de género en la participación laboral femenina. Serie Documentos de Trabajo 374. Departamento de Economía, Universidad de Chile.

Contreras, D., Otero, G., Díaz, J. y Suárez, N. 2019. Inequality in Social Capital in Chile: Assessing the Importance of Network Size and Contacts' Occupational Prestige on Status Attainment. Social Networks 58, 59-77.

Cronbach, L. 1951. Coefficient Alpha and the Internal Structure of Tests. Psychometrika 16(3), 297-334.

Delattre, E. y Sabatier, M. 2007. Social Capital and Wages: An Econometric Evaluation of Social Networking's Effects. Labour 21(2), 209-236.

DiMaggio, P. y Garip, F. 2012. Network Effects and Social Inequality. Annual Review of Sociology 38, 93-118.

Fitzenberger, B. y Wunderlich, G. 2003. Gender Wage Differentials in West Germany: A Cohort Analysis. German Economic Review 3(4), 379-414.

Fuentes, A. y Vergara, R. 2018a. Brecha salarial de género: evolución en el período 1990-2017. Puntos de Referencia 490. Disponible en: https://www.cepchile.cl/ cep/site/artic/20181013/asocfile/20181013082029/pder490_afuentes_rvergara. pdf [19 noviembre 2019].

Fuentes, A. y Vergara, R. 2018b. The Public Sector Wage Premium and Its Evolution in the Period 1990-2017. Estudios Públicos 152, 7-45.

Fuentes, J., Palma, A. y Montero, R. 2005. Discriminación salarial por género en Chile: una mirada global. Estudios de Economía 32(2), 133-157.

García, J., Hernández, P. y López-Nicolás, Á. 2001. How Wide is the Gap? An Investigation of Gender Wage Differences Using Quantile Regression. Empirical Economics 26(1), 149-167.

Gardeazabal, J. y Ugidos, A. 2005. Gender Wage Discrimination at Quantiles. Journal of Population Economics 18(1), 165-179.

Granovetter, M. 1973. The Strength of Weak Ties. American Journal of Sociology 78(6), 1360-1380. 
Granovetter, M. 1974. Getting a Job: A Study of Contacts and Careers. Chicago: University of Chicago Press.

Granovetter, M. 1983. The Strength of Weak Ties: A Networks Theory Revisited. Sociological Theory 1, 201-233.

Heckman, J. 1979. Sample Selection Bias as a Specification Error. Econometrica 47(1), 153-161.

Heckman, J. 1980. Sample Selection Bias as a Specification Error with an Application to the Estimation of Labor Supply Functions (206-248). En Smith, J. (ed.), Female Labor Supply: Theory and Estimation. New York: Princeton University Press.

Horvarth, G. 2014. Occupational Mismatch and Social Networks. Journal of Economic Behavior \& Organization 106, 442-468.

Jáuregui, A. 2016. Efectos de la discriminación salarial por género en pobreza y desigualdad. Tesis Mg Políticas Públicas, Repositorio Académico, Universidad de Chile.

Koenker, R. y Bassett, G. 1978. Regression Quantiles. Econometrica 46(1), 33-50.

Koenker, R. y D'Orey, V. 1987. Computing Regression Quantiles. Journal of the Royal Statistical Society 36(3), 383-393.

Lin, N. 1995. Les ressources sociales: une théorie du capital social. Revue francaise de sociologie 36(4), 685-704.

Lin, N. 1999. Social Networks and Status Attainment. Annual Review of Sociology 25, 467-487.

Lin, N. 2001. Social Capital. A Theory of Social Structure and Action. Cambridge: Cambridge University Press.

Long, W., Appleton, S. y Song, L. 2017. The Impact of Job Contacts Networks on Wages of Rural-Urban Migrants in China: A Switching Regression Approach. Journal of Chinese Economic and Business Studies 15, 81-101.

Luke, N. y Munshi, K. 2006. New Roles for Marriage in Urban Africa: Kinship Networks and the Labor Market in Kenya. The Review of Economics and Statistics 88(2), 264-282.

Melly, B. 2005. Public-Private Sector Wage Differentials in Germany: Evidence from Quantile Regression. Empirical Economics 30(2), 505-502.

Merton, R. 1968. The Matthew Effect in Science. Science 159(3810), 56-63.

Mincer, J. 1962. Labor Force Participation of Married Women: A Study of Labor Supply (63-97). En Lewis, H.G. (ed.), Aspects of Labor Economics. New York: Princeton University Press.

Mincer, J. 1974. Schooling, Experience, and Earnings. New York: National Bureau of Economic Research, Columbia University Press.

Montgomery, J. 1991. Social Networks and Labor-Market Outcomes: Toward an Economic Analysis. The American Economic Review 81(5), 1408-1418.

Montgomery, J. 1994. Weak Ties, Employment, and Inequality: An Equilibrium Analysis. American Journal of Sociology 99(5), 1212-1236. 
Mood, C. 2010. Logistic Regression: Why We Cannot Do what We Think We Can Do, and what We Can Do about It. European Sociological Review 26(1), 6782.

Moore, S. y Kawachi, I. 2017. Twenty Years of Social Capital and Health Research: A Glossary. Journal of Epidemiology \& Community Health 71(5), 513-517.

Mora, C. y Blanco, O. 2017. The Gender Deficit: Everyday Practices of Differentiation in the Chilean Labour Market. Bulletin of Latin American Research 37(3), 291-305.

Mortensen, D. y Vishwanath, T. 1994. Personal Contacts and Earnings: It is Who You Know! Labour Economics 1(2), 187-201.

Mouw, T. 2003. Social Capital and Finding a Job: Do Contacts Matter? American Sociological Review 68(6), 868-898.

Mueller, R. 1998. Public-Private Sector Wage Differentials in Canada: Evidence from Quantile Regressions. Economic Letters 60, 229-235.

Munshi, K. 2003. Networks in the Modern Economy: Mexican Immigrants in the U.S. Labor Market. The Quarterly Journal of Economics 118(2), 549-599.

Observatorio Social 2016. Metodología de medición de pobreza multidimensional con entorno y redes. Serie Documentos Metodológicos Casen 32. Ministerio de Desarrollo Social, Gobierno de Chile.

Perticará, M. y Bueno, I. 2009. Brechas salariales por género en Chile: un nuevo enfoque. Revista Cepal 99, 133-149.

Petersen, T., Saporta, I. y Seidel, M.D. 2000. Offering a Job: Meritocracy and Social Networks. American Journal of Sociology 106(3), 763-816.

Portes, A. 1998. Social Capital: Its Origins and Applications in Modern Sociology. Annual Review of Sociology 24(1), 1-24.

Portes, A. y Sensenbrenner, J. 1993. Embeddedness and Immigration: Notes on the Social Determinants of Economic Action. American Journal of Sociology 86, 1320-1350.

Rees, A. 1966. Information Networks in Labor Markets. American Economic Association 56(2), 559-566.

Rees, A. y Schultz, G. 1970. Workers in an Urban Labor Market. Chicago: Chicago University Press.

Schurch, R. 2013. El retorno de las carreras: un estudio de caso de los factores que inciden en las remuneraciones de universitarios recién titulados. Calidad en la Educación 38, 215-244.

Stoloff, J., Glanville, J. y Bienenstock, E. 1999. Women's Participation in the Labor Force: The Role of Social Networks. Social Networks 21, 91-108.

Stupnytska, Y. y Zaharieva, A. 2017. Optimal Policy and the Role of Social Contacts in a Search Model with Heterogeneous Workers. Journal of Public Economic Theory 19(5), 957-985.

Sylos Labini, M. 2005. Social Networks and Wages: It Is All about Connections! LEM Working Paper Series 2004/10. 
Tardos, R. 1996. Some Remarks on the Interpretation of the 'Social Capital' Concept with Special Regard to the Hungarian Case. Bulletin of Sociological Methodology 53, 52-62.

Uehara, E. 1990. Dual Exchange Theory, Social Networks, and Informal Social Support. American Journal of Sociology 96(3), 521-557.

van der Gaag, M. y Snijders, T. 2005. The Resource Generator: Social Capital Quantification with Concrete Items. Social Networks 27, 1-29.

Völker, B. y Flap, H. 1999. Getting ahead in the GDR: Social Capital and Status Attainment under Communism. Acta Sociologica 42(1), 17-34.

Williams, R. 2009. Using Heterogeneous Choice Models to Compare Logit and Probit Coefficients across Groups. Sociological Methods \& Research 37(4), 531-559. EP 